\title{
Uma Abordagem para Gestão de Incidentes em Ecossistemas de Software Proprietário
}

\author{
Luiz Alexandre M. Costa ${ }^{1}$, Awdren Fontão ${ }^{2}$ (coorientador), Rodrigo Santos ${ }^{1}$ (orientador) \\ ${ }^{1}$ Programa de Pós-Graduação em Informática \\ Universidade Federal do Estado do Rio de Janeiro (UNIRIO) \\ Rio de Janeiro - RJ - Brasil \\ ${ }^{2}$ Programa de Pós-graduação em Ciência da Computação \\ Universidade Federal do Mato Grosso do Sul (UFMS) \\ Campo Grande - MS - Brasil \\ luiz.costa@edu.unirio.br, awdren@facom.ufms.br, rps@uniriotec.br
}

\begin{abstract}
Systems unavailability that makes up a proprietary software ecosystem (SECO) platform, those that are protected by intellectual property, hinders productivity and revenue. It also damages SECO platform and the organization's image. In this scenario, maintaining a technological platform mitigating the risks of incidents (unplanned service interruption) is one of the existing activities to support SECO. This work aims to develop and evaluate an approach based on an incident management process in order to support the IT management team in the evaluation of the proprietary SECO platform maintenance.
\end{abstract}

Resumo. A indisponibilidade de sistemas que compõem uma plataforma de ecossistema de software (ECOS) proprietário, aqueles que são protegidos pela propriedade intelectual, prejudica produtividade e receita, além de provocar danos ao ECOS e à imagem da organização. Neste cenário, manter uma plataforma tecnológica mitigando os riscos de incidentes (interrupção não planejada de um serviço) é uma das atividades existentes para sustentar o ECOS. Este trabalho tem como objetivo desenvolver e avaliar uma abordagem baseada em um processo para gestão de incidentes visando apoiar a equipe de gerenciamento de TI na avaliação da manuteção da plataforma de um ECOS proprietário.

\section{Introdução}

O desenvolvimento de um produto de software único tem sido substituído por uma estratégia onde múltiplos softwares se integram por meio de uma plataforma tecnológica comum [Van Den Berk et al. 2010] [Santos 2017], criando Ecossistemas de Software (ECOS). O contexto deste trabalho está em torno de ECOS proprietário, isto é, aquele constituído de contribuições protegidas por propriedade intelectual [Manikas 2016]. Neste caso um conjunto de atores se relacionam como fornecedores, desenvolvedores internos, colaboradores externos e gestores de TI, competindo e cooperando entre si como, por exemplo, ecossistemas de comércio eletrônico.

Há estudos que apontam que várias causas de indisponibilidade de sistemas (i.e., desastres naturais, falhas humanas, violações de segurança) pertencentes ou não a um 
ECOS proprietário, prejudicam a produtividade e a receita de uma organização, além de provocar danos à imagem e reputação, conforme apontado por Creeden et al. (2013). Manter uma plataforma mitigando os riscos de incidentes (i.e., interrupção não planejada de um serviço ou a redução da sua qualidade) é uma das atividades existentes para sustentar um ECOS e pode ser uma oportunidade para resolver os desafios que estão além dos problemas técnicos: as preocupações de negócios e de perspectiva social [Sadi e Yu 2015].

Ainda nessa conjuntura, um dos atores do ECOS, especialmente os gestores de TI, possuem papel importante, pois são eles os responsáveis pelo acompanhamento e gerenciamento dos ativos de software da plataforma, integrando-os às necessidades estratégicas da companhia, como otimização de processos e aumento da produtividade [Sumner et al. 2006]. Logo, agir rapidamente no momento em que um incidente ocorre no ambiente produtivo de algum dos sistemas mantidos pela organização central pode ser um fator competitivo para a empresa. Torna-se, então, mais um desafio para que os gestores de TI mantenham sempre disponíveis os sistemas que suportam a operação do negócio, permitindo o seu crescimento [Mansur 2007].

Este trabalho tem como objetivo geral desenvolver e avaliar uma abordagem baseada em um processo para gestão de incidentes visando apoiar a equipe de gerenciamento de TI na avaliação da manuteção da plataforma de um ECOS proprietário. Portanto, uma visão estruturada deste trabalho baseia-se em: (i) apresentar um corpo de conhecimento sobre o tema; (ii) idealizar um processo de gestão de incidentes em ECOS proprietário que permita conhecimento à equipe de gestão de TI; e (iii) avaliar a originalidade e o valor da contribuição.

\section{Caracterização do Problema}

De acordo com o estudo de Manikas (2016), para as classificações de ECOS (aberto, híbrido e proprietário), não há muitas pesquisas em ECOS proprietário, também em virtude da dificuldade de acesso a dados desses ambientes. Além disso, por meio de uma revisão adhoc na literatura de artigos científicos em ECOS proprietário, notou-se uma falta de estudos que investiguem o contexto de gestão de incidentes.

A indisponibilidade de algum ativo de software (i.e., sistema, aplicação, serviço ou componente) que compõe a plataforma tecnológica comum de um ECOS proprietário é um fator que afeta os lucros da organização, mas que pode ser evitado. Por isso, as causas de indisponibilidade devem ser uma preocupação constante da equipe de gerenciamento de TI, responsável por manter a organização central. Tais incidentes, isto é, interrupções não planejadas dos ativos de software, podem comprometer as respectivas empresas, seja em imagem ou financeiramente [Creeden et al. 2013].

A gestão de incidentes é uma atividade que tem como principal objetivo restaurar a operação normal do serviço o mais rápido possível, minimizando os prejuízos à operação do negócio e garantindo assim o melhor nível de serviço e disponibilidade [Cusick e Ma 2010]. Um ECOS pode enfrentar dificuldades na falta ou na deficiência de um processo de gestão de incidentes, causando desequilíbrio nas relações internas e externas em relação à sua plataforma [Luciano et al. 2012]. Além disso, o pouco conhecimento para tomada de decisão deixa de proporcionar à organização um diagnóstico dos incidentes para avaliar manutenções na plataforma do ECOS. 


\section{Proposta de Solução}

Para atingir o objetivo geral desta pesquisa, pretende-se alcançar os seguintes objetivos específicos: i) definir um corpo de conhecimento sobre a relevância de mecanismos de governança de ativos de software em ECOS proprietário; ii) definir um corpo de conhecimento sobre gestão de incidentes em ECOS proprietário; iii) estabelecer um processo para gestão de incidentes em ECOS proprietário; e iv) identificar mecanismos de monitoramento para diagnóstico de incidentes em ECOS proprietário a partir de dados de sua plataforma e comunidade (apoio ferramental).

\section{Metodologia}

A metodologia de pesquisa utilizada neste trabalho é composta por duas fases: concepção, na qual o foco é a definição e construção da abordagem para gestão de incidentes em ECOS proprietário; e avaliação, focada em avaliar a viabilidade e a utilização da abordagem. Na fase de concepção, visando avaliar os fatores que influenciam o processo de gestão de incidentes a partir da lente teórica de ECOS proprietário, serão realizados um estudo exploratório, um mapeamento sistemático da literatura e uma pesquisa de opinião com especialistas. Na fase de avaliação, será realizado um estudo de viabilidade para verificar a aplicabilidade do artefato em um caso real de um ECOS proprietário.

\section{Avaliação da Solução}

Para avaliar a solução, serão executados estudos experimentais em uma organização líder na indústria brasileira de seguros, visando verificar a abordagem proposta em relação ao seu objetivo [Shull et al. 2001]. Neste trabalho, será realizado um estudo de viabilidade onde os participantes serão divididos em dois grupos, equilibrados a partir do perfil de caracterização. A investigação ocorrerá de forma que cada grupo cumpra um conjunto de tarefas: um deles utilizará o processo instanciado por meio da ferramenta de suporte à abordagem proposta e o outro utilizará as ferramentas disponíveis na empresa. Ao final, os participantes serão convidados a preencher um questionário de avaliação da tecnologia utilizando um modelo TAM $^{1}$ na escala NPS ${ }^{2}$. Após a etapa de avaliação, uma etapa de refinamento é indicada, com o objetivo de atuar nos ajustes identificados a partir dos resultados parciais obtidos.

\section{Atividades Realizadas}

Dentre as atividades propostas para concepção e avaliação da solução, realizou-se um estudo exploratório com os objetivos de capturar percepções sobre mecanismos de governança em um ECOS proprietário e se familiarizar com o fenômeno que está sendo investigado. Foram realizadas uma pesquisa de opinião e entrevistas com gestores de TI. Por meio de uma análise de correlação, identificou-se os mecanismos de maior relevância e as influências desses gestores. Este estudo deu origem ao artigo "Investigating Asset Governance Mechanisms in a Proprietary Software Ecosystem”, aceito para publicação e apresentação na Trilha Principal do SBSI 2020 [Costa et al. 2020]. Um mapeamento sistemático está em andamento visando identificar estudos sobre gestão de incidentes no contexto de ECOS.

\footnotetext{
${ }^{1}$ Technology Acceptance Model é um modelo de avaliação utilizado em sistemas de informação que analisa como os usuários passam a aceitar e usar uma tecnologia.

${ }^{2}$ Net Promoter Score é uma métrica de lealdade do cliente, que traz reflexos de experiência e satisfação.
} 


\section{Considerações Finais}

Este trabalho apresentou um projeto de pesquisa de dissertação cujas principais contribuções são: uma análise de governança de ativos de software em ECOS proprietário; e fornecimento de bases de informações, processo e ferramenta relacionados aos conceitos de gestão de incidentes em ECOS proprietário.

\section{Referências}

Costa, L. A. M., Fontão, A., and Santos, R. P. (2020). Investigating asset governance mechanisms in a proprietary software ecosystem. In XVI Brazilian Symposium on Information Systems.

Creeden, D. M., Glionna, J., Poulter, M. C., Kaptinski, J. S., Persico, J. R., Doolittle, W. R., Cascade, R. S., Van Heyst, A. J., Ernst, D. A., Chomienne, K. M., et al. (2013). Methods and systems for managing risk management information. US Patent $8,589,273$.

Cusick, J. J. and Ma, G. (2010). Creating an itil inspired incident management approach: Roots, response, and results. In 2010 IEEE/IFIP Network Operations and Management Symposium Workshops, pages 142-148. IEEE.

Luciano, E. M., Testa, M. G., and de Azevedo Bragança, C. E. B. (2012). Percebendo os benefícios e dificuldades da adoção da gestão de serviços de tecnologia da informação. REGE-Revista de Gestão, 19(1):145-164.

Manikas, K. (2016). Revisiting software ecosystems research: A longitudinal literature study. Journal of Systems and Software, 117:84-103.

Mansur, R. (2007). Governança de TI: metodologias, frameworks e melhores práticas. Brasport.

Sadi, M. H. and Yu, E. (2015). Designing software ecosystems: How can modeling techniques help? In Enterprise, Business-Process and Information Systems Modeling, pages 360-375. Springer.

Santos, R. (2017). Ecossistemas de software no projeto e desenvolvimento de plataformas para jogos e entretenimento digital. Anais do XVI Simpósio Brasileiro de Jogos e Entretenimento Digital, pages 2-4.

Shull, F., Carver, J., and Travassos, G. H. (2001). An empirical methodology for introducing software processes. ACM SIGSOFT Software Engineering Notes, 26(5):288-296.

Sumner, M., Bock, D., and Giamartino, G. (2006). Exploring the linkage between the characteristics of it project leaders and project success. Information systems management, 23(4):43-49.

Van Den Berk, I., Jansen, S., and Luinenburg, L. (2010). Software ecosystems: a software ecosystem strategy assessment model. In Proceedings of the Fourth European Conference on Software Architecture: Companion Volume, pages 127-134. 